\title{
Descrição de duas novas técnicas cirúrgicas para o tratamento de prolapso vaginal em vacas zebuínas: vaginectomia parcial e vaginopexia dorsal
}

\author{
Description of two new surgical techniques for the treatment of vaginal \\ prolapse in zebuine cows: partial vaginectomy and dorsal vaginopexy
}

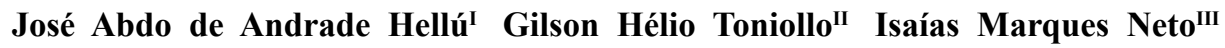

\section{RESUMO}

\begin{abstract}
Com o aumento da casuística e o insucesso da aplicação das técnicas convencionais de Caslick, Bühner ou Flessa, na redução do prolapso vaginal não associado à gestação em vacas zebuínas, caracterizada pela elevação significativa da recorrência da afecção, este trabalho teve por objetivo avaliar duas novas técnicas cirúrgicas na correção do prolapso vaginal, denominadas de vaginectomia parcial e vaginopexia dorsal em vacas. $O$ estudo foi conduzido a campo, por um periodo de quatro anos, utilizando-se 812 vacas zebuinas (Nelore, Gir e Brahman), em idade reprodutiva, alojadas em diversas propriedades. O diagnóstico foi realizado através de anamnese e avaliação dos sinais clínicos e, de acordo com o estágio do prolapso vaginal, foi definida a técnica cirúrgica a ser executada, após procedimentos anestésicos. Os resultados pós-cirúrgicos das duas técnicas indicaram alta porcentagem de recuperação (93,4\% para vaginectomia parcial e $96,14 \%$ para vaginopexia dorsal), baixo indice de recidivas (6,3\% e 3,7\%, respectivamente) e baixa mortalidade (entre $0,2 \%$ e $0,3 \%$ ). Desse modo, os resultados demonstram que as duas técnicas propostas podem ser indicadas para a redução e solução definitiva de prolapso vaginal em vacas zebuínas.
\end{abstract}

Palavras-chave: prolapso, vaca, vaginectomia, vaginopexia e vaginal.

\section{ABSTRACT}

Vaginal prolapse is an increasing problem in nonpregnant zebuine cows that so far conventional surgical techniques such as Caslick, Buhner and Flessa have failed to replace. This study aimed to assess the use of two new techniques, partial vaginectomy and dorsal vaginopexy, for the replacement of the prolapsed vagina in 812 adult zebuine cows (nelore, gir and brahman breeds) from several farms during a four years period. The selection of the technique to be used was based on clinical history, physical examination and stage of the vaginal prolapse. Post-surgical results indicated a high recovery percentage for partial vaginectomy and dorsal vaginopexy $(93.4 \%$ and $96.1 \%$, respectively), low relapse rate (6.3\% and $3.7 \%$, respectively) and low mortality (between $0.2 \%$ and $0.3 \%$, respectively). Altogether, these findings showed that both techniques can be used for the effective reduction of vaginal prolapse in non-pregnant zebuine cows.

Key words: prolapse, cow, vaginectomy, vaginopexy and vaginal.

\section{INTRODUÇÃO}

Entre as principais afecções vaginais dos animais domésticos, incluem-se a inversão e o prolapso vaginal (BERCHTOLD, 1988; SAH \& NAKAO, 2003; ALVARENGA, 2006), que são descritos na literatura como afecções dos ruminantes, associadas ao terço final da gestação e ao puerpério, quando podem estar associadas ao prolapso uterino (BERCHTOLD, 1988; NASCIMENTO \& SANTOS, 2003).

É designado prolapso vaginal a saída da parede do órgão através da vulva, podendo ser de maior ou menor grau. A etiologia da afecção está relacionada ao aumento do estrógeno circulante nas últimas semanas da gestação, o que induz o relaxamento dos ligamentos pélvicos e perineais, associado ao aumento uterino gravídico, facilitando o prolapso (BERCHTOLD, 1988; NASCIMENTO \& SANTOS, 2003; JACKSON, 2004; ALVARENGA, 2006).

$\mathrm{O}$ prolapso é classificado em grau 1, grau 2, grau 3 e grau 4 (SAH \& NAKAO, 2003; ALVARENGA, 2006; DIAS, 2007), dependendo da evolução do problema. No primeiro estágio, ocorre protusão da mucosa vaginal através da vulva, quando

'Programa de Pós-graduação em Ciências Agrárias e Veterinárias, Universidade Paulista “Dr. Júlio de Mesquita Filho” (UNESP), Campus de Jaboticabal, 14401-600, São Paulo, SP, Brasil. E-mail: ja@jasaudeanimal.com.br. Autor para correspondência.

"Departamento de Medicina Veterinária Preventiva e Reprodução Animal, Universidade Paulista "Dr. Júlio de Mesquita Filho" (UNESP), São Paulo, SP, Brasil.

IIIFarmacêutico da Empresa J.A. Saúde Animal, Franca, SP, Brasil. 
o animal está em decúbito, desaparecendo quando este se levanta. Já no segundo, a protusão da mucosa vaginal permanece mesmo com o animal na posição quadrupedal. Em seguida, no terceiro estágio, o prolapso vaginal e cervical permanece constantemente exposto e, no último estágio (grau 4), apresenta-se com áreas necrosadas (ALVARENGA, 2006).

Os sinais clínicos mais observados são a exposição parcial ou total da vagina pela rima vulvar, inquietação, lesões da porção evertida de leve a grave, dissolução parcial ou total do tampão mucoso, retenção urinária, prolapso retal secundário ao tenesmo, congestão venosa passiva com consequente desvitalização da estrutura prolapsada, vulvite, vaginite, cervicite e, nas fêmeas gestantes, é possível observar abortamento ou morte fetal por contaminação com enfisema fetal (TONIOLLO \& VICENTE, 2003; DIAS, 2007).

Geralmente, o aparecimento dessa afecção ocorre em animais de alto valor comercial e, desse modo, foram descritas tentativas de redução do prolapso, utilizando-se as técnicas de Caslick, Bühner ou Flessa, porém, sem o sucesso esperado, devido à recorrência após breve ou longo período após a retirada dos meios de contenção (TONIOLLO \& VICENTE, 2003; RICHARD, 2007; PRESTES et al., 2008). Considerando-se essas afirmativas e tendo ciência da casuística da afecção nos bovinos, entende-se a necessidade da proposição de novos tratamentos na correção definitiva da afecção.

O objetivo deste trabalho foi avaliar duas novas técnicas cirúrgicas na correção do prolapso vaginal, denominadas vaginectomia parcial e vaginopexia dorsal em vacas.

\section{MATERIAL E MÉTODOS}

O estudo foi conduzido a campo, por um período de quatro anos (abril/2008 a maio/2012), utilizando-se 812 vacas zebuínas (Nelore, Gir e Brahman), na idade reprodutiva, alojadas em diversas propriedades, de acordo com a casuística, assim distribuídas: 366 vacas no Estado de Minas Gerais (45,07\%), 325 no Estado de São Paulo (40,02\%), 25 no Estado do Rio de Janeiro (3,07\%), 24 no Estado de Goiás (2,96\%), 24 no Estado do Mato Grosso (2,96\%), 24 no Estado do Mato Grosso do Sul (2,96\%) e 24 no Estado do Paraná (2,96\%).

Os animais que apresentaram a afecção e os parâmetros clínicos dentro da faixa de normalidade foram selecionados. O diagnóstico do prolapso vaginal foi realizado por anamnese e avaliação dos sinais clínicos apresentados, diferenciando de ruptura vaginal, hérnia perineal, prolapso de bexiga, cistos das glândulas de Bartholin, hematoma de vulva e de neoplasias, cujos sinais são semelhantes, sendo os prolapsos classificados em grau 1, grau 2 e grau 3 (Figura 1A, B, C) e, de acordo com o estágio, foi definida a técnica cirúrgica a ser executada.

Os animais foram submetidos a jejum alimentar e hídrico por 12 horas. Em ambas as técnicas, foi realizada anestesia peridural intercoccígea com a associação de lidocaína e xilazina (Bloc ${ }^{\circledR}$ - Produtos Veterinários J.A. - Patrocínio Paulista - SP - Brasil). Assim, introduziu-se uma agulha longa (Cateter Intravenoso Nipro ${ }^{\circledR} 14$ G Sorocaba - SP - Brasil) entre a primeira e a segunda vértebras coccígeas, depositando-se 4 a $5 \mathrm{~mL}$ do produto, equivalente a $0,2 \mathrm{mg} \mathrm{kg}^{-1}$ de lidocaína e $0,004 \mathrm{mg} \mathrm{kg}^{-1}$ de xilazina. $\mathrm{Na}$ técnica de vaginopexia dorsal, além da anestesia peridural intercoccígea, nos dois locais da fixação, sobre a região do músculo glúteo médio e do músculo gluteobíceps, foram utilizados dois bloqueios anestésicos com $10 \mathrm{~mL}$ do mesmo produto, aplicandose na musculatura, $5 \mathrm{~mL}$ superficialmente e $5 \mathrm{~mL}$ profundamente, com auxílio de seringa (Seringa descartável estéril 20mL BD Plastipak ${ }^{\circledR}$ São Paulo -

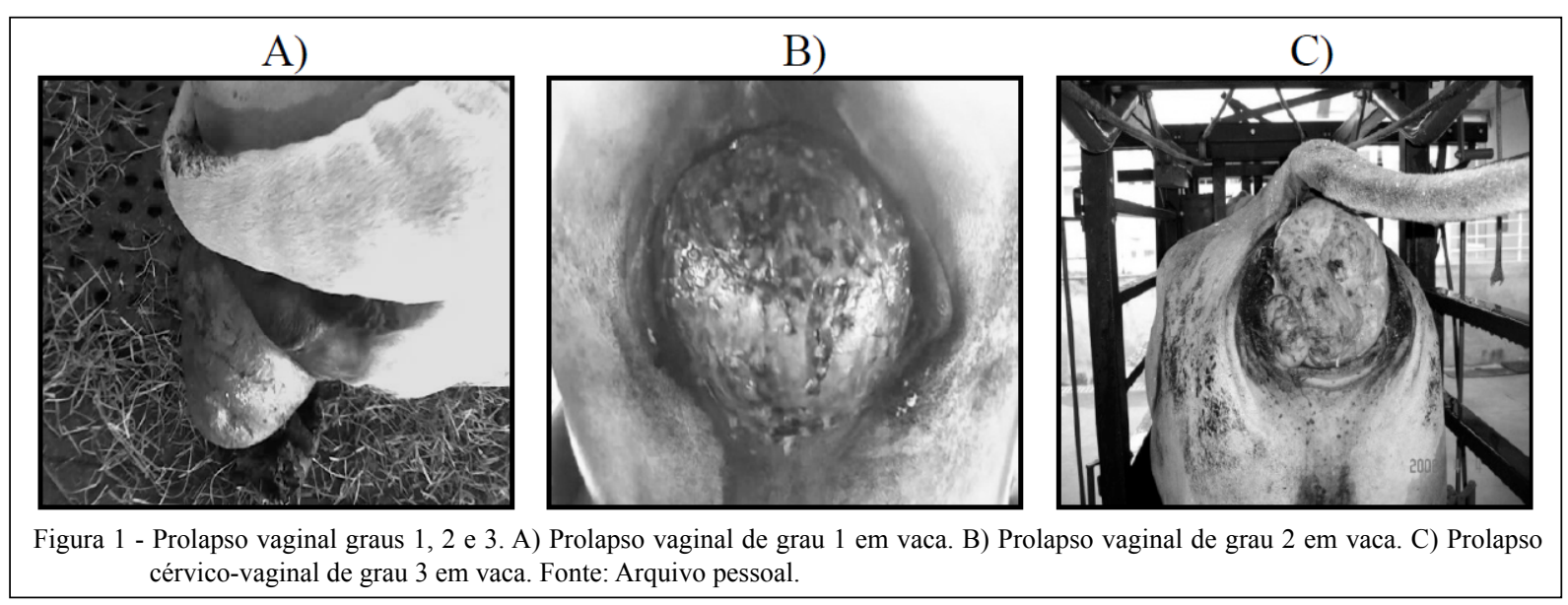

Ciência Rural, v.45, n.11, nov, 2015. 
SP - Brasil) e agulha (Agulha estéril 40 x 12 BD PrecisionGlide ${ }^{\mathrm{TM}}$ São Paulo - SP - Brasil).

A técnica de vaginectomia parcial destinouse ao tratamento do prolapso vaginal de grau 1. Para isso, fez-se a identificação da porção da vagina prolapsada, que se apresenta com textura áspera e geralmente localizada lateralmente. Com o auxílio de duas ou três pinças de Kocher, exteriorizou-se o tecido vaginal acometido e realizou-se a antissepsia da mucosa com iodo povidona (PVPI Degermante 10\% Vansi ${ }^{\circledR}$ - Vansil Saúde Animal - Descalvado - SP Brasil). Com uma pinça de conchectomia, o tecido a ser retirado foi fixado o mais próximo possível da mucosa vaginal íntegra, evitando o pinçamento do meato urinário e da uretra (Figura 2). Realizouse uma sutura no tecido íntegro próximo à pinça, no sentido cranial (Figura 3A), utilizando um padrão interrompido tipo Wolff, mantendo-se a extremidade do fio de cada ponto para efetuar o próximo.

Foi empregado o fio absorvível, composto de ácido poliglicólico trançado $\mathrm{n}^{\circ} 2$ (Brasuture - São Sebastião da Grama - SP - Brasil). Com bisturi elétrico (Modelo B-1.001 Deltronix Equipamentos ${ }^{\circledR}$ São Paulo SP - Brasil), caudalmente à pinça de conchectomia, retirou-se todo o tecido acometido (Figura 3B) e realizou-se a aproximação da parede vaginal incisada com uma sutura contínua festonada, utilizando fio categute cromado $\mathrm{n}^{\mathrm{o}} 4$ (Brasuture - São Sebastião da Grama - SP - Brasil) (Figura 3C). Finalmente, a pinça de conchectomia foi removida, reposicionando a mucosa vaginal. A inexistência de sangramento e a integridade do meato urinário foram avaliadas (Figura 3D).

A vaginopexia dorsal foi conduzida para o tratamento do prolapso de $2^{\circ}$ e de $3^{\circ}$ graus, nos quais a vagina e a cérvix do útero estavam prolapsados. Realizou-se a antissepsia do períneo com iodo

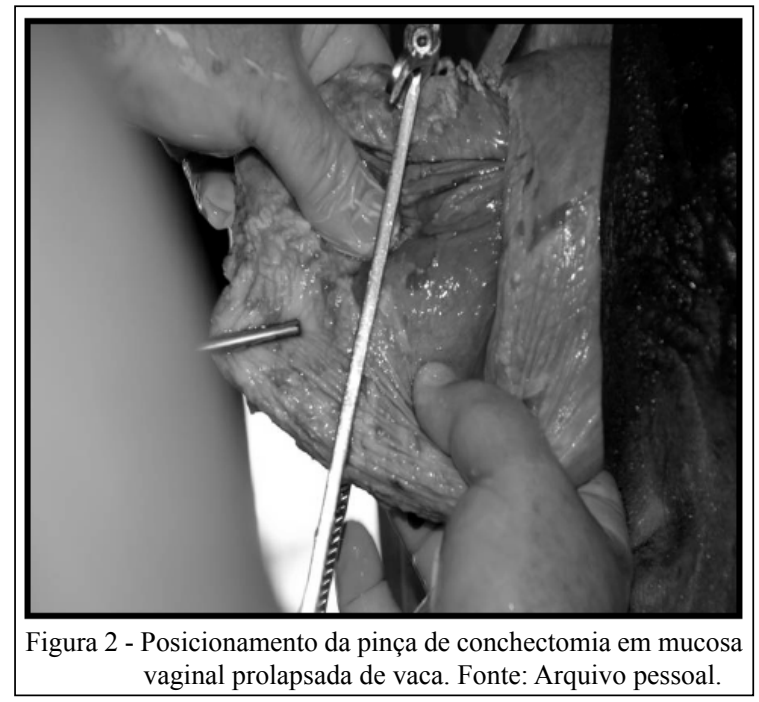

povidona (PVPI Degermante 10\% Vansil ${ }^{\circledR}$ - Vansil Saúde Animal -Descalvado - SP - Brasil). Logo após a execução do bloqueio anestésico peridural intercoccígeo, a porção prolapsada foi reposicionada e se definiu em qual dos lados da pelve deveria ser realizada a vaginopexia.

Foi introduzido um espéculo vaginal tubular no reto do animal (Figura 4A), fixandose ao ânus com sutura em bolsa de fumo, com fio de náilon $\mathrm{n}^{\circ} 2$ (Brasuture - São Sebastião da Grama - SP Brasil). Esse procedimento permitiu a localização e deslocamento do reto no momento da sutura vaginal, evitando a sua transfixação.

Após ampla tricotomia e antissepsia da região lateral glútea escolhida para a vaginopexia (Figura 4B, C), com uma das mãos no interior da vagina, localizaram-se os dois pontos de fixação, sendo um na parte cranial da parede dorsal da pelve, próximo ao osso sacro, evitando-se o plexo sacral (região menos espessa da parede), e outro na parte caudal da parede dorsal da pelve, sobre a borda caudal do ligamento sacrotuberal, evitando-se a artéria ilíaca interna (Figura 4D). Realizou-se o bloqueio anestésico nos dois pontos, injetando-se, com o auxílio de seringa (seringa descartável estéril 20mL BD Plastipak ${ }^{\circledR}$ São Paulo - SP - Brasil) e agulha (agulha estéril 40 x 12 BD PrecisionGlide $^{\mathrm{TM}}$ São Paulo - SP - Brasil), $5 \mathrm{~mL}$ de uma associação de lidocaína e xilazina (Bloc ${ }^{\circledR}$ - Produtos Veterinários J.A. - Patrocínio Paulista - SP - Brasil) na região subcutânea e $5 \mathrm{~mL}$ mais profundamente, na musculatura, ligamento sacrotuberal e parede vaginal. Com um bisturi, duas incisões foram realizadas na pele, paralelas e distantes aproximadamente $5 \mathrm{~cm}$ das bordas laterais das vértebras sacrais e caudais, medindo aproximadamente $5 \mathrm{~cm}$ de comprimento e distando em torno de $15 \mathrm{~cm}$ uma da outra (Figura 4E). Introduziu-se pela vagina uma abraçadeira de náilon (Alumbra - 400 x 7,2mm - São Bernardo do Campo SP - Brasil), medindo $40 \mathrm{~cm}$ de comprimento, 7,2mm de largura e $1,0 \mathrm{~mm}$ de espessura.

O reto foi deslocado para o lado oposto e a palma da mão foi posicionada no interior da vagina, pressionando a parede vaginal dorsal contra o ligamento sacrotuberal. Introduziu-se uma agulha do tipo Gerlach, com aproximadamente $30 \mathrm{~cm}$ de comprimento, na comissura cranial da primeira incisão, orientando-a perpendicularmente através da musculatura e do ligamento sacrotuberal, até alcançar o lúmen vaginal, onde foi introduzida a extremidade da abraçadeira no orifício da agulha e tracionada para o exterior, ficando esta posicionada com a extremidade basal no lúmen da vagina e a apical no exterior da parede dorsal da pelve (Figura 4F, G, H, I).

Do lado externo, a ponta da agulha foi posicionada na comissura caudal a $5 \mathrm{~cm}$ do local de 


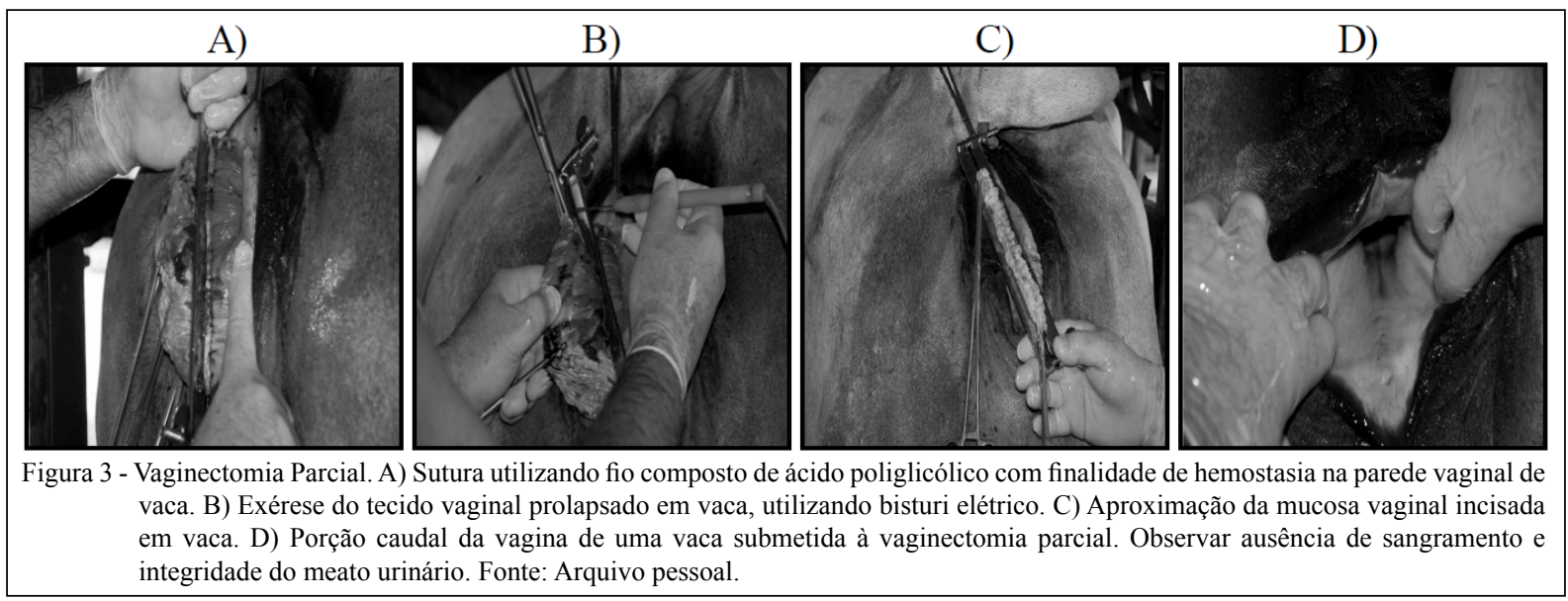

inserção e novamente pressionada perpendicularmente até atingir o lúmen vaginal. No interior da vagina, as duas extremidades da abraçadeira foram aproximadas, cerradas e a ponta excedente cortada (Figura 4J).

Em seguida, o mesmo procedimento foi repetido caudalmente na região glútea, precisamente sobre a borda caudal do ligamento sacrotuberal. As duas incisões da pele foram aproximadas com pontos simples separados, utilizando-se fio de náilon $\mathrm{n}^{\circ} 2$ (Brasuture - São Sebastião da Grama SP - Brasil), deixando as abraçadeiras sepultadas sob a pele (Figura 4K). O espéculo tubular foi retirado por palpação. Sobre as feridas cirúrgicas, foi aplicado cicatrizante-repelente, a base de Clorpirifós, Diclorvós e Violeta Genciana (Lepecid BR Spray ${ }^{\circledR}$ Ouro Fino Agronegócio - Cravinhos - SP - Brasil).

No período pós-operatório, em ambas as técnicas, foram administradas uma associação de cloridrato de enrofloxacina e diclofenaco de sódio, (Diclotril $^{\circledR}$ - Produtos Veterinários J.A. - Patrocínio Paulista - SP - Brasil), na dose de $1 \mathrm{~mL}$ para cada $40 \mathrm{~kg}$ de peso corporal, o que corresponde a $2,5 \mathrm{mg}$ de cloridrato de enrofloxacina e $0,9375 \mathrm{mg}$ de diclofenaco de sódio por quilo de peso vivo, pela via intramuscular, a cada 24 horas, por sete dias.

$\mathrm{Na}$ técnica da vaginopexia dorsal, os pontos da pele foram retirados 10 dias após o procedimento cirúrgico e as abraçadeiras permaneceram por um período mínimo de 90 dias. Nos casos em que foi observada a fistulação dorsal ou secreção vaginal purulenta, as abraçadeiras foram retiradas após os 90 dias, por meio de tração e corte pelo lúmen da vagina (Figura 5), utilizando-se uma pinça hemostática e bisturi.

\section{RESULTADOS E DISCUSSÃO}

No diagnóstico clínico dos prolapsos vaginais de 812 vacas zebuínas, visualizou-se: 39,41\% de animais em $1^{\circ}$ estágio ou grau $1 ; 47,66 \%$ de animais em $2^{\circ}$ estágio ou grau 2; e $12,93 \%$ em $3^{\circ}$ estágio ou grau 3. Embora a literatura pesquisada não mencione a incidência dessa afecção em bovinos não gestantes, PRESTES et al. (2008) relatam que se trata de uma nova afecção da reprodução. Nos animais submetidos à vaginectomia parcial ou à vaginopexia dorsal, havia sido realizada a aspiração folicular e constatou-se que não estavam prenhes e apresentavam-se obesas. Essas observações corroboram quanto à origem multifatorial da afecção, incluindo o acesso à vagina repetidamente pela punção ovariana para colheita de oócitos (PRESTES et al., 2008).

Os animais que apresentaram prolapso vaginal de grau 1 foram submetidos à cirurgia pela técnica da vaginectomia parcial. Os resultados pósoperatórios obtidos para a técnica de vaginectomia foram: $93,44 \%$ de animais recuperados; $6,25 \%$ de animais apresentando recidivas; e 0,31\% de animais que foram a óbitos. ALVARENGA (2006) relata que, na maior parte dos casos de prolapsos que ocorrem durante a gestação, os sinais desaparecem após o parto, mas recidivam no próximo período gestacional. No entanto, nesses casos de não gestantes, as recidivas foram observadas em períodos variáveis após a cirurgia.

Um dos pontos críticos na realização da técnica da vaginectomia parcial é evitar hemorragia, pela fixação da pinça de conchectomia e a realização da sutura cranialmente a esta, que devem ser realizadas de forma cuidadosa, evitando que vasos de maior calibre fiquem sem hemostasia. Na técnica descrita por Farquharson, em 1949 (ARTHUR et al., 2001), a hemorragia também é mencionada como crítica no momento da ressecção da submucosa. Para evitar a hemorragia, descreve-se que sejam realizadas dissecções circunferenciais em segmentos distintos.

Outro cuidado a ser tomado durante a realização da vaginectomia parcial é a não transfixação 


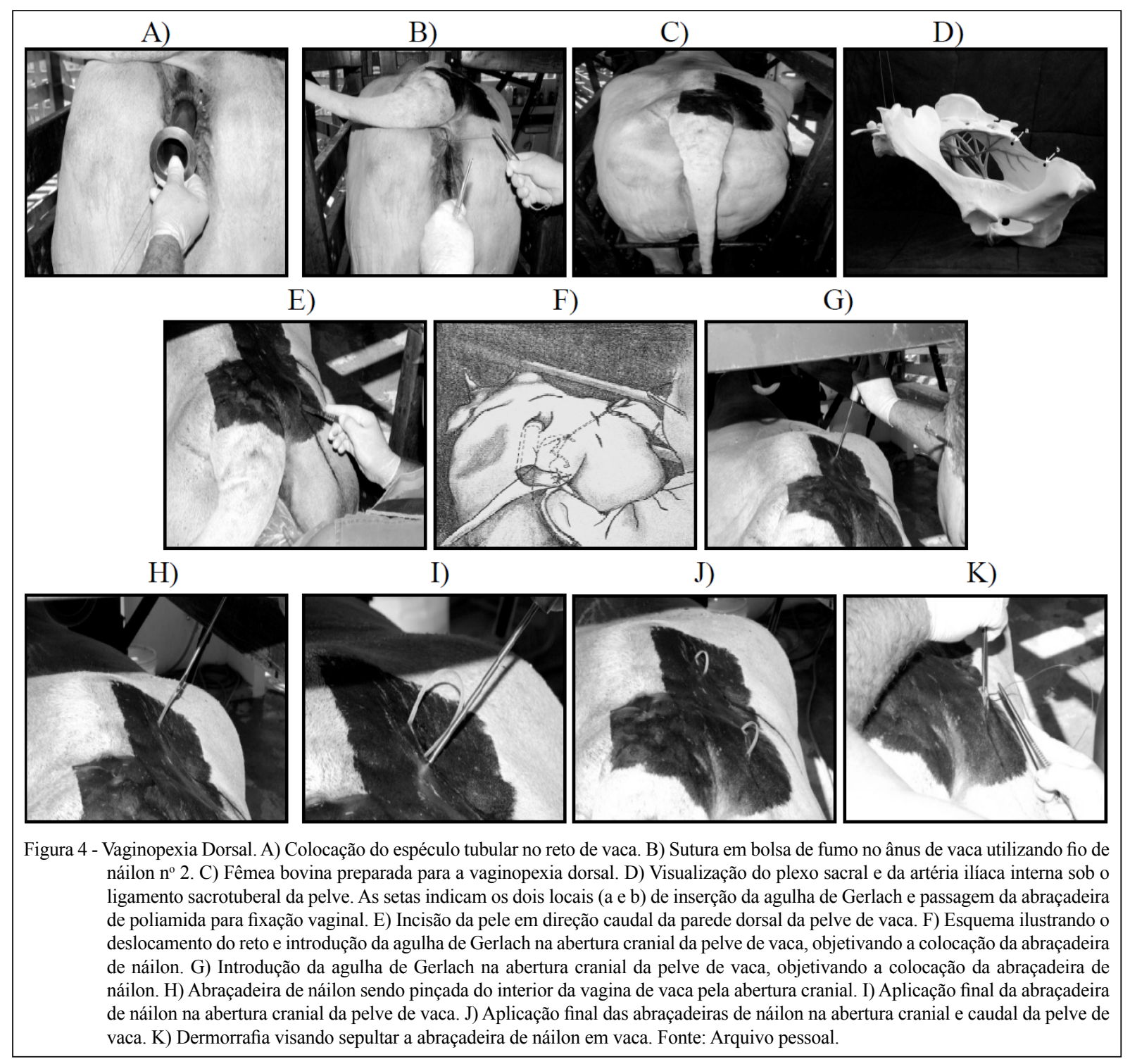

da uretra ou do meato urinário. Sobre esse fato, apenas foi encontrada referência dos autores TONIOLLO \& VICENTE (2003), quando mencionam o tratamento

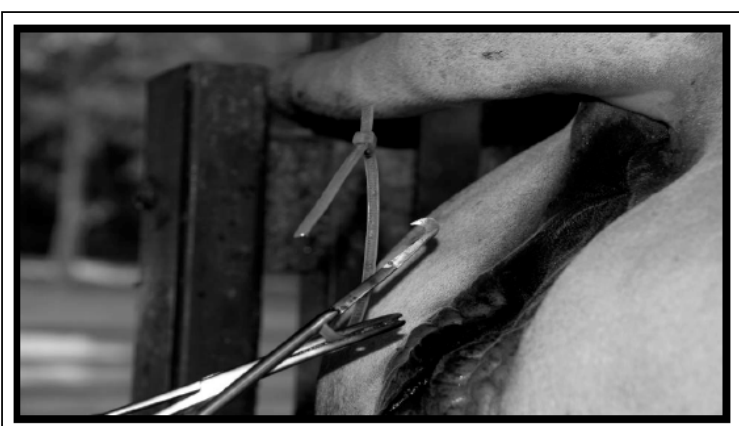

Figura 5 - Retirada da abraçadeira de náilon após 90 dias. Fonte: Arquivo pessoal. de prolapso vaginal utilizando fixação da vagina no tendão pré-púbico, cujo maior risco é a ligadura da uretra e a lesão da bexiga.

RICHARD (2007) também menciona este tipo de complicação na técnica cervicopexia de Winkler. No momento da fixação da pinça de conchectomia, na porção da parede vaginal a ser amputada, evitou-se que o meato urinário fosse preso por ela, com esse cuidado não ocorreu nenhum caso de transfixação da uretra ou do meato urinário.

A técnica denominada vaginopexia dorsal foi idealizada sem o conhecimento dos conceitos da técnica de Minchev (RICHARD, 2007) ou do botão de Johnson (GARNERO \& PERUSIA, 2006), as quais preconizam a fixação da vagina prolapsada no ligamento sacrociático e aos músculos glúteos, pelo 
fato de estas não serem citadas na maioria dos livros didáticos disponíveis e não serem utilizadas na rotina.

Diferentemente das duas técnicas citadas, na vaginopexia dorsal empregada neste experimento, não se utiliza nenhum tipo de botão, pino ou outro objeto equivalente aparente na parte externa dos animais, fazendo uso apenas de abraçadeiras de náilon. Outra diferença entre as técnicas é que, na vaginopexia dorsal, nenhum tipo de instrumento fica exposto na região da garupa, eliminando o risco de se tornar um ponto de entrada para infecções e comprometer a estética do animal.

A técnica foi aplicada em 492 animais, sendo 387 para a correção de prolapso vaginal de $2^{\circ}$ estágio e 105 para correção de prolapso vaginal de $3^{\circ}$ estágio. Nos casos mais graves, foi realizado tratamento prévio a base de higienização diária e medicação parenteral por 15 dias para diminuir a inflamação dos tecidos, facilitando o reposicionamento do prolapso.

Em um caso em que o animal apresentava concomitantemente prolapso vaginal e retal (Figura 6), foi necessária a realização da amputação da porção prolapsada do reto pela técnica descrita por BERGE \& WESTHUES (1975), redução temporária do prolapso vaginal pela técnica de Bühner, terapia antimicrobiana e anti-inflamatória por 15 dias, para posterior aplicação da vaginopexia dorsal.

Os resultados pós-cirúrgicos obtidos para a técnica de vaginopexia dorsal em vacas zebuínas foram de $96,1 \%$ de animais recuperados; 3,7\% de animais apresentando recidivas; e $0,20 \%$ de animais que foram a óbitos. Os animais que apresentaram recidivas passaram por avaliação clínica, decidindo-se pela realização de uma nova cirurgia, utilizando a mesma técnica, desta vez fixando a vagina do outro lado da pelve, promovendo a recuperação destes. Tal fato é relatado por PRESTES et al. (2008), quando se utiliza das técnicas convencionais de Caslick, Bühner ou Flessa.

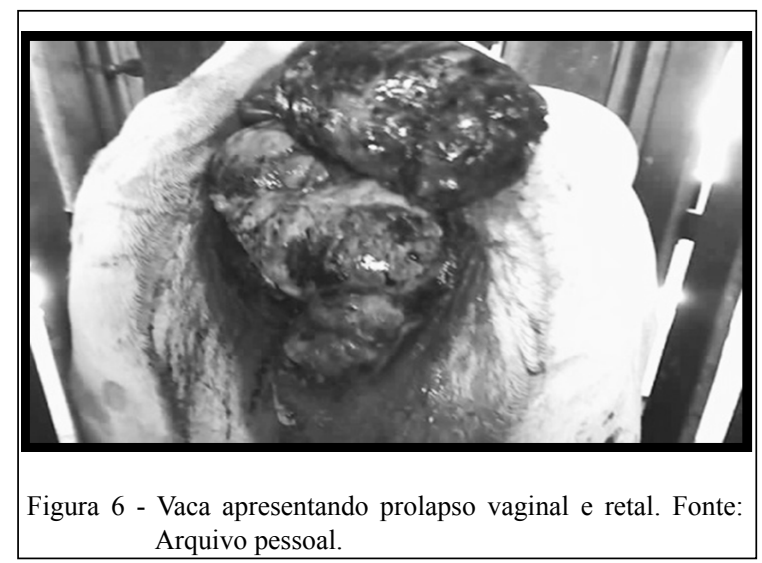

$\mathrm{O}$ único animal que veio a óbito com a aplicação dessa técnica, onde foi aplicada a vaginopexia dorsal nos dois lados da pelve, no mesmo procedimento cirúrgico, na necropsia, foi verificado acúmulo de fezes no reto, indicando interrupção do trânsito intestinal, provavelmente, causada pela dor provocada pela compressão do reto e distensão da vagina, sendo então este procedimento não mais aplicado.

Em 93 vacas, nas quais a técnica de vaginopexia dorsal foi empregada (18,9\%), observou-se o aparecimento de fístulas nos locais da fixação da abraçadeira, sendo 17,2\% com drenagem externa e $82,8 \%$ no lúmen da vagina. Nesses casos, as abraçadeiras foram retiradas após a permanência mínima de noventa dias pós-cirurgia, tempo suficiente para que houvesse fibrosamento local e aderência da parede da vagina à parede dorsal da pelve.

No presente trabalho, foram obtidos índices de mortalidade de $0,31 \%$ e de $0,20 \%$, respectivamente, nas vacas tratadas com a técnica da vaginectomia parcial e nas vacas tratadas com a técnica vaginopexia dorsal.

\section{CONCLUSÃO}

A vaginectomia parcial é uma técnica de fácil execução e eficiente para o tratamento dos prolapsos vaginais de grau 1, com raras complicações; a vaginopexia dorsal é uma técnica de dificuldade mediana, contudo viável para o tratamento dos prolapsos vaginais em estágios mais avançados, com mínimas complicações. Os resultados demonstram que as duas técnicas são indicadas para a redução e solução definitiva de prolapso vaginal em vacas.

\section{COMITÊ DE ÉTICA}

Certificamos que o protocolo n. 21.861/10 da pesquisa intitulada está de acordo com os Princípios Éticos na Experimentação Animal, adotado pelo Colégio Brasileiro de Experimentação (COBEA) e aprovado pela Comissão Ética no uso de Animais (CEUA). Reunião ordinária, 22 out. 2010.

\section{REFERÊNCIAS}

ARTHUR, G. et al. Prolapse of the vagina and cervix. In: Veterinary reproduction and obstetrics. 8.ed. London: Elsevier, 2001. p.144-152.

ALVARENGA, F.C.L. Patologias da gestação. In: PRESTES, N.C.; ALVARENGA, F.C.L. Obstetrícia veterinária. Rio de Janeiro: Guanabara Koogan, 2006. p.149-155.

BERCHTOLD, M. Enfermedades de la vagina, vestibule y vulva. In: GRUNERT, E.; BERCHTOLD, M. Infertilidad en la vaca. Buenos Aires: Hemisferio Sur, 1988. p.261-276. 
BERGE, E.; WESTHUES, M. Operaciones en tórax, abdômen y cola. In: __ Técnica operatoria veterinaria. 5.ed. Barcelona: Labor, 1975. Cap.E, p.278-280.

DIAS, B.M.L. Clínica das espécies pecuárias e cirurgias corretivas. 2007. 60f. Relatório final de estágio (Licenciatura em Medicina Veterinária) - Universidade de Trás-os-Montes e Alta Douro, Vila Real.

GARNERO, O.; PERUSIA, O. Aparelho reprodutor da fêmea. In: Manual de anestesia e cirurgia de bovinos. São Paulo: Tecmed, 2006. p.59-76.

JACKSON, P.G.G. Vaginal prolapse. In: Handbook of veterinary obstetric. 2.ed. China: Elsevier, 2004. p.21-23;216-217.

NASCIMENTO, E.F.; SANTOS, R.L. Patologias do cérvix, da vagina e da vulva. In: Patologia da reprodução dos animais domésticos. $\overline{2 . e d .}$ Rio de Janeiro: Guanabara Koogan, 2003. p.84-89.
PRESTES, N.C. et al. Prolapso total ou parcial de vagina em vacas não gestantes: uma nova modalidade de patologia? Revista Brasileira de Reprodução Animal, v.32, n.3, p.182-190, jul./set. 2008. Disponível em: <http://www.cbra.org.br/pages/publicações/ $\mathrm{rbra} /$ download/RB 181\%20Prestes\%20vr3\%20pag182-190.pdf $>$.

RICHARD, M.H. Surgical correction of abnormalities of genital organs of cows. In: YOUNGQUIST, R.S.; THRELFALL, W.R. Current therapy in large animal theriogenology. 2.ed. bMissouri: Saunders Elsevier, 2007.b p.463-472. Disponível em: <http://www. sciencedirect.com/science/article/pii/B9780721693231500635>. Acesso em: 07 nov. 2011. doi: 10.1016/B978-072169323-1.50063-5.

SAH, S.K.; NAKAO, T.b Some characteristics of vaginal prolapse in nepali buffaloes. Journal of Veterinary Medical Science, v.65, n.11, p.1213-1215, nov. 2003. Disponível em: <https://www. jstage.jst.go.jp/article/jvms/65/11/65_11_1213/_pdf>. Acesso em: 08 nov. 2011. doi: $10.1292 /$ jvms. $65 . \overline{12} 1 \overline{3}$.

TONIOLLO, G.H.; VICENTE, W.R.R. Patologias da gestação. In: Manual de obstetrícia veterinária. São Paulo: Varela, 2003. Cap.V, p.43-64. 


\section{ERRATA}

Artigo "Descrição de duas novas técnicas cirúrgicas para o tratamento de prolapso vaginal em vacas zebuínas: vaginectomia parcial e vaginopexia dorsal" publicado no fascículo v45n11 de novembro da Ciência Rural páginas 2026-2032 sem a inclusão de dois autores, onde se lia:

"José Abdo de Andrade Hellú́l"

IPrograma de Pós-graduação em Ciências Agrárias e Veterinárias, Universidade Paulista “Dr. Júlio de Mesquita Filho” (UNESP), Campus de Jaboticabal, 14401-600, São Paulo, SP, Brasil. E-mail: ja@jasaudeanimal.com.br. Autor para correspondência.

leia-se:

"José Abdo de Andrade Hellú ${ }^{\mathrm{I}}$ Gilson Hélio Toniollo ${ }^{\mathrm{II}}$ Isaías Marques Neto"III"

IPrograma de Pós-graduação em Ciências Agrárias e Veterinárias, Universidade Paulista “Dr. Júlio de Mesquita Filho” (UNESP), Campus de Jaboticabal, 14401-600, São Paulo, SP, Brasil. E-mail: ja@jasaudeanimal.com.br. Autor para correspondência.

"Departamento de Medicina Veterinária Preventiva e Reprodução Animal, Universidade Paulista "Dr. Júlio de Mesquita Filho" (UNESP), São Paulo, SP, Brasil.

IIIFarmacêutico da Empresa J.A. Saúde Animal, Franca, SP, Brasil.

Para a versão correta, acesse:

http://www.scielo.br/pdf/cr/v45n11/1678-4596-cr-0103_8478cr20140528.pdf 